\title{
Modelización técnico-ecónomica de un sistema de moldeo de aluminio a alta presión para fabricación de piezas de automoción ${ }^{(\bullet)}$
}

\author{
F. Faura ${ }^{(*)}$ \\ Resumen En el presente trabajo se desarrolla un modelo técnico-económico para un sistema de fundición \\ inyectada a alta presión de aleaciones de aluminio. Para la obtención de los datos necesarios en las \\ estimaciones y correlaciones utilizadas por el modelo se han analizado los sistemas de producción de \\ empresas que utilizan estos procesos. Esto ha permitido determinar las variables tecnológicas que \\ afectan de manera más relevante a los aspectos económicos del proceso. Se ha desarrollado una apli- \\ cación informática que permite explorar fácilmente la influencia de distintos parámetros del sistema. \\ Palabras clave: Modelos técnico-económicos. Fundición de aluminio. Fundición a alta presión.

\section{Technical-economic modelling of an aluminum high pressure die casting system for automotive parts fabrication}

\begin{abstract}
In the present paper a technical-economic model for an aluminum high pressure die casting system has been developed. In order to obtain the necessary data for the correlations utilized by the model, has been analyzed the production systems of companies that use these processes. This has allowed to determine the most important technological variables that affect to the economical aspect of the process. A computer application has been developed which allows to explore easily the influence of different system parameters.
\end{abstract}

Keywords: Technical economic models. Aluminum die casting. Die casting.

\section{INTRODUCCIÓN}

Uno de los mayores retos en el campo de la ingeniería de procesos de materiales es integrar las consideraciones técnicas, económicas y medioambientales, de forma razonada y eficiente. Hasta ahora no existen fórmulas claramente definidas para dirigir estos problemas, por lo que las decisiones carecen, en la mayoría de los casos con demasiada frecuencia, de bases técnico-económicas (1).

Los modelos matemáticos, en general, constituyen una herramienta muy adecuada para conseguir estos objetivos. Estas aplicaciones cubren un amplio espectro que abarca, por una parte, desde la modelización de simples átomos o pequeñas agrupaciones atómicas, hasta la instalación de un proceso completo de materiales, y por otra, desde el análisis puramente científico de alguna característica

$(\bullet$ Trabajo recibido el día 23 de septiembre de 1996.

(*) Dpto. de Ingeniería de Materiales y Fabricación. E.T.S. Ingenieros Industriales. Universidad de Murcia. Alfonso XIII, 34. 30203-Cartagena (España). del material hasta los aspectos de un carácter estrictamente económico del mismo. En particular, los modelos técnico-económicos, se presentan como una de las metodologías más adecuadas para analizar aspectos de los sistemas materiales, tales como la competitividad entre distintas tecnologías de procesamiento, el impacto de la introducción de una nueva tecnología o el estudio de fenómenos de sustitución de materiales (2-5).

La procesos de fundición inyectada han sido ampliamente aplicados en la industria de automoción, en particular las instalaciones de cámara fría, las cuales son una de las más empleadas para la fabricación de componentes de aluminio. La aplicación de estos procesos a estos entornos productivos ha hecho que se desarrollen distintas tecnologías e incluso que estén emergiendo actualmente otras, que compiten en este dinámico sector (6).

Los consumos actuales y previsiones a corto y medio plazo de las aleaciones de aluminio en el sector son muy alentadoras ( 7 y 8). Las características básicas de estos materiales les hacen muy atractivos para satisfacer algunos de los objetivos prioritarios de esta industria y sus consumidores, como 
son los relacionados con el medio ambiente (menor consumo energético, reciclado, etc.).

Los principales problemas que presenta la fundición inyectada son los relacionados con las, relativamente bajas, características mecánicas de los productos. Esto se debe, fundamentalmente, a la porosidad de las piezas obtenidas por inyección a alta presión (9).

Las nuevas y emergentes tecnologías que tratan de atenuar estas deficiencias son muy variadas. Algunas de las más relevantes son las tecnologías: thixocasting, rheocasting, vacural casting, NDC (New Die Casting), o squeeze casting. Todas ellas mejoran sustancialmente las características mecánicas y porosidades en las piezas fabricadas.

En el presente trabajo se desarrolla un modelo técnico-económico para un sistema de fundición inyectada a alta presión que pueda servir de base para su posterior adaptación a las particularidades de las distintas tecnologías que están compitiendo en el mercado. Mediante la aplicación informática desarrollada, el modelo puede ser utilizado de forma eficiente para explorar los parámetros clave del sistema. El modelo forma parte de un macromodelo de análisis de sistemas materiales que está actualmente desarrollándose en el Materials Processing Center, MIT.

\section{CARACTERÍSTICAS BÁSICAS DEL SISTEMA}

La fundición inyectada a alta presión es un proceso de fabricación tecnológicamente maduro, obteniéndose con él una amplia diversidad de piezas con excelente calidad dimensional (tolerancias lineales de $\pm 0,3 \%$ en aleaciones de aluminio) y unas características mecánicas razonables (10). Como resultado de la excelente precisión dimensional y de la calidad superficial de las piezas, la mayoría de estas no requieren mecanizados adicionales salvo un desbarbado ligero y, quizá, operaciones de taladrado y/o roscado de orificios.

En la tecnología de cámara fría, se pueden alcanzar presiones y velocidades de inyección del orden de $1.000 \mathrm{~kg} / \mathrm{cm}^{2}$ y $6 \mathrm{~m} / \mathrm{s}$, respectivamente, existiendo en el mercado máquinas de hasta $5.000 \mathrm{t}$ de fuerza de cierre del molde. La productividad es elevada debido al fuerte grado de automatización existente y, además, los moldes pueden producir muchos miles de piezas sin cambios dimensionales importantes.

Desde el punto de vista técnico, el sistema de producción puede presentar muy diversas etapas en la ruta del proceso, desde la fusión del material hasta la expedición del producto obtenido. La primera etapa del procesado incluiría las fases de: fusión y mantenimiento a la temperatura de colada, inyección del metal fundido, extracción y desbarbado de la pieza. El postprocesado de la pieza después de su extracción puede incluir procesos tales como tratamientos térmicos, mecanizado, ensayos no destructivos, control dimensional y/o geométrico, etc. El estudio efectuado se ha centrado en la primera etapa (proceso de fundición) no considerando el resto de operaciones de la ruta del proceso.

En cada uno de los pasos o fases de esta etapa es posible aplicar la metodología general de los modelos técnico-económicos para análisis de sistemas materiales (11). De esta forma se puede disponer de un modelo que permite explorar, entre otros aspectos, la influencia de los parámetros claves del proceso en los costes del mismo.

Puesto que el proceso está fuertemente automatizado, algunas etapas claramente diferenciadas, en la ruta del proceso, pueden considerarse agrupadas desde el punto de vista de la construcción del modelo. Esta ha sido la opción considerada con respecto a las dos últimas fases o pasos de la etapa analizada.

La sección de fusión y mantenimiento del metal a la temperatura necesaria, se ha considerado como un subsistema que puede ser utilizado por todas, o parte de las máquinas de la instalación, por lo que afectará con una fracción de sus costes al análisis de cada máquina. El molde utilizado en la máquina de inyección se considerará como una entrada que se agrega al flujo económico del proceso de fabricación en el apartado de herramientas, ya que éste no suele ser fabricado en las instalaciones de la fundición sino que se adquiere directamente del exterior.

La máquina o instalación de inyección propiamente dicha es la más importante desde el punto de vista del modelo, ya que constituye el núcleo fundamental de la instalación, formando la denominada línea de producción. En este subsistema se encuentra la máquina o máquinas principales que recogen el metal fundido del subsistema de fusión y mantenimiento, inyectándolo en el molde. Este subsistema está constituido por dos partes: depósitos servidores y máquina de inyección. En último lugar se tiene la cadena de extracción y desbarbado (automática o manual). En esta sección se eliminan canales de llenado y mazarotas de la pieza recién extraída del molde quedando la pieza preparada para la etapa de postprocesado.

\section{MODELO TÉCNICO-ECONÓMICO DESA- RROLLADO}

De acuerdo con la estructura general de los modelos técnico-económicos (Fig. 1), se dispone de un primer módulo de introducción de datos que se divide en dos secciones: introducción de datos propiamente dicha y bloque de estimación, donde se 


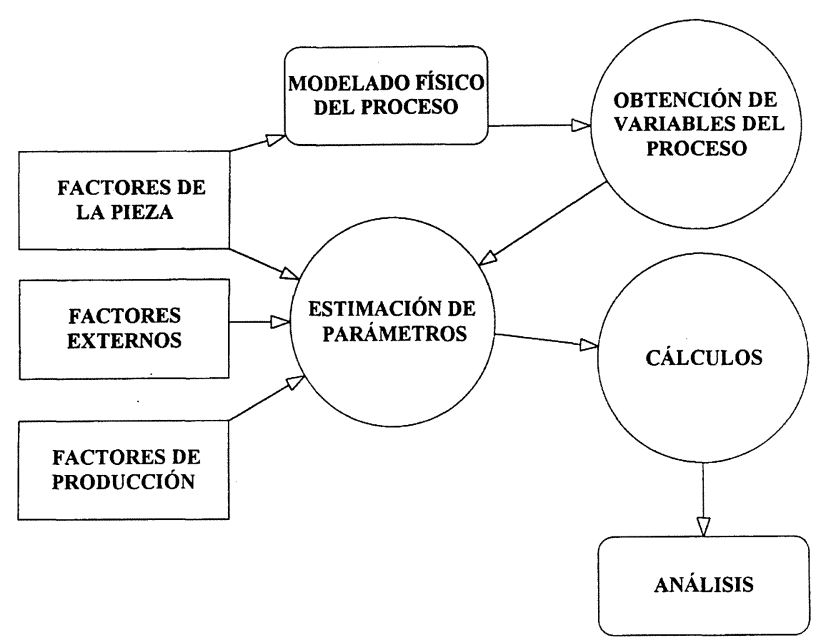

Fig. 1. - Diagrama de flujo de datos de un modelo técnico-económico.

FIG. 1.-Flowchart of a technical-economic model.

interrelacionan diferentes datos de entrada. Esta última sección es una de las partes más delicadas de la modelización, ya que es en ella donde se estiman datos de gran incidencia en el estudio económico. Siguiendo el flujo de información se entra en el módulo en donde se realizan los cálculos que permiten obtener datos relativos a costes, bienes de equipo, líneas de producción necesarias, etc. Por último, en el bloque de análisis se analiza la sensibilidad experimentada por ciertos resultados del sistema ante perturbaciones provocadas en las entradas. Así, es posible conocer, por ejemplo, cómo afecta la presión de trabajo al coste de maquinaria, al coste de producción total de la pieza, etc.

En la figura 1 aparecen, junto a los principales bloques de introducción de datos, otros bloques como el de simulación física del proceso, que son complementarios al modelo técnico-económico (TCM), pero que por pertenecer a otro ámbito del análisis del proceso, sólo se tendrán en cuenta como generadores de ciertos datos de entrada.

El seguimiento del flujo de información del modelo puede hacerse a través de la aplicación informática desarrollada.

La implementación de la aplicación ha sido realizada en lenguaje Microsoft Visual Basic 3.0, el cual permite una programación orientada a objetos y dirigida por eventos. El sistema gestor de bases de datos ha sido Microsoft Access 2.0.

La mayoría de los TCM descritos en la bibliografía siguen un flujo lineal, no considerando aspectos tales como el reciclado y/o la reelaboración del material en la línea de producción. Los efectos introducidos por los mismos, no linealidad en el flujo principal del proceso, pueden observarse en las figuras 2 y 3.

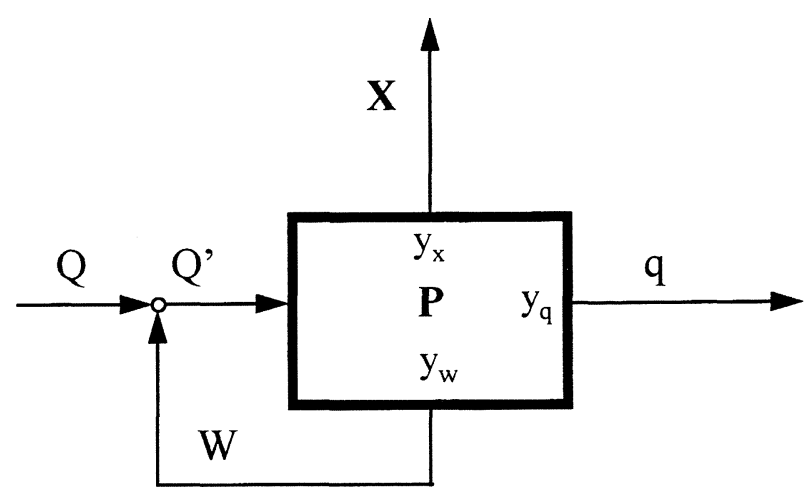

FIG. 2.- Representación gráfica de un bucle simple (salida reciclable).

FIG. 2.- Schematic of the simple arrow loop (recyclable output).

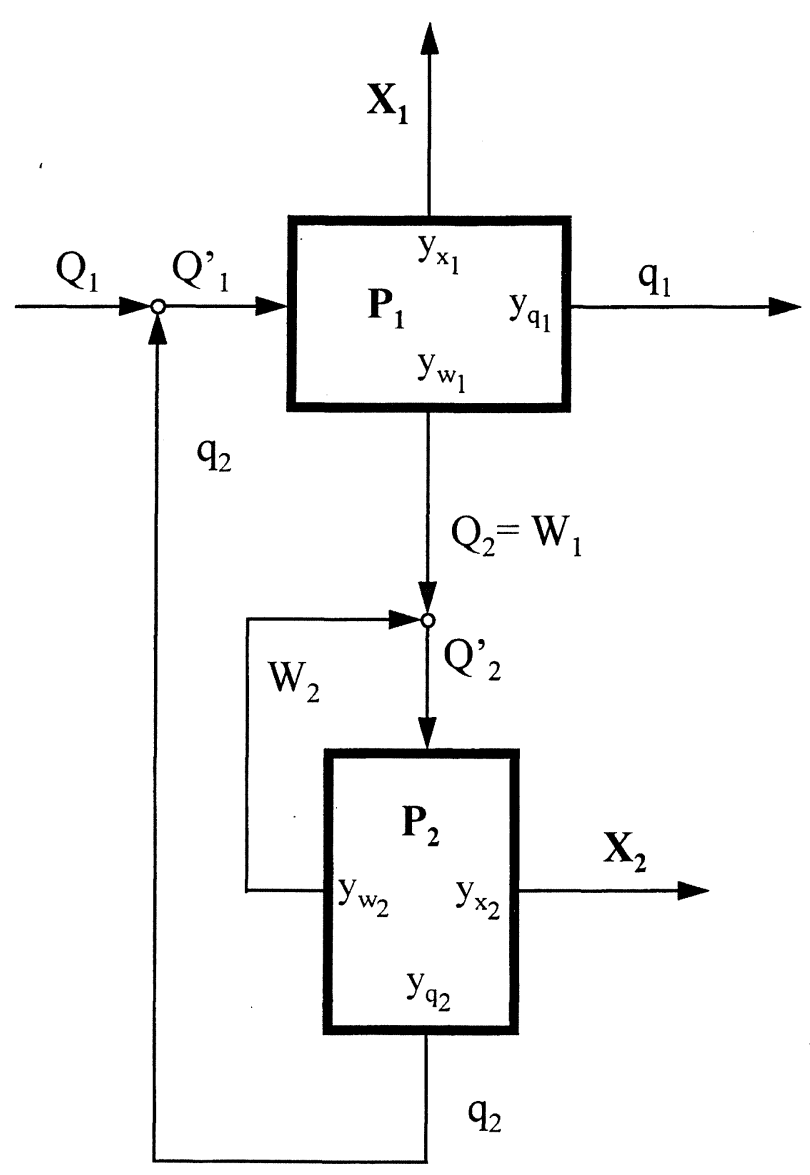

FIG. 3.- Representación gráfica de un bucle con procesado (salida con estación de reelaboración).

FIG. 3.- Schematic of the processing step loop (rework station).

Con objeto de considerar la incidencia de los mismos en el sistema, se han introducido en el modelo formulaciones matemáticas sencillas que permiten cuantificar los efectos sobre el material de la presencia de estos bucles. Estos cálculos son compatibles con el concepto de "cálculos lineales 
inversos del volumen de producción objetivo" (11), habitualmente utilizados en los TCM.

\subsection{Bucles de reciclado y reelaboración}

Se han considerado dos casos, bucles simples (simple arrow loops), en los que la salida es reciclable directamente, y bucles con procesado (processing step loops), en los que se incluyen estaciones de reelaboración.

En los primeros (Fig. 2), suponiendo un proceso de producción que esté dividido en $n$ pasos o fases, la salida reciclable en el primer paso del proceso sería: $y_{\mathrm{w}} Q$; y en el paso $n: y_{\mathrm{w}}^{\mathrm{n}} Q$. Por lo tanto, en el paso $P$ del proceso la entrada real será:

$$
Q^{\prime}=\sum_{\mathrm{i}=0}^{\infty} Q \cdot y_{\mathrm{w}}^{\mathrm{i}}=Q \sum_{\mathrm{i}=0}^{\infty} y_{\mathrm{w}}^{\mathrm{i}}=\frac{Q}{1-y_{\mathrm{w}}}
$$

y por tanto:

$$
\begin{gathered}
q=y_{\mathrm{p}} \cdot Q^{\prime}=\frac{y_{\mathrm{p}}}{1-y_{\mathrm{w}}} \cdot Q \\
Q=\frac{1-y_{\mathrm{w}}}{y_{\mathrm{p}}} \cdot q
\end{gathered}
$$

Estas ecuaciones pueden ser utilizadas, por ejemplo, en el reciclado continuo de materiales en la etapa de preparación del material donde no son necesarios etapas de reelaboración específica.

En el segundo caso (Fig. 3), utilizando el mismo planteamiento que en el caso anterior, en el paso $n$, la salida reciclable en $P_{1}$ sería: $y_{\mathrm{w} 1} q_{2}^{\mathrm{n}-1}$ y en $P_{2}$ generaría la cantidad: $q_{2}^{\mathrm{n}}$ a ser reciclada. De los cálculos del caso anterior: ${ }^{2}$

$$
q_{2}^{\mathrm{n}}=\left(\frac{y_{\mathrm{p} 2}}{1-y_{\mathrm{w} 2}} y_{\mathrm{wi}}\right)^{n} \cdot Q_{1}
$$

Por tanto, el paso del proceso $P$ tendrá en realidad la entrada:

$$
Q_{1}^{\prime}=\sum_{\mathrm{i}=0}^{\infty}\left(\frac{y_{\mathrm{p} 2}}{1-y_{\mathrm{w} 2}} \cdot y_{\mathrm{w} 1}\right)^{\mathrm{i}} \cdot Q_{1}=\frac{1-y_{\mathrm{w} 2}}{1-y_{\mathrm{w} 2}-y_{\mathrm{p} 2} y_{\mathrm{p} 1}} \cdot Q_{1}
$$

puesto que

$$
q_{1}=y_{\mathrm{p} 1} \cdot Q_{1}^{\prime}
$$

entonces

$$
Q_{1}=\frac{1-y_{\mathrm{w} 2}-y_{\mathrm{p} 2} y_{\mathrm{w} 1}}{y_{\mathrm{p} 1}-y_{\mathrm{w} 2} y_{\mathrm{p} 1}} \cdot q_{1}
$$

Es importante notar que un bucle con procesado puede ser asociado con un flujo lineal equivalente de rendimiento efectivo:

$$
y_{\text {leff }}=\frac{y_{\mathrm{p} 1}-y_{\mathrm{w} 2} y_{\mathrm{p} 1}}{1-y_{\mathrm{w} 2}-y_{\mathrm{p} 2} y_{\mathrm{w} 1}}
$$

Este caso asume que una salida reciclable puede ser procesada un número infinito de veces en $P_{2}$. En el caso práctico de una estación de reelaboración es posible que la práctica industrial imponga un número finito de pasos $n$ en dicha estación. En esas condiciones, la ecuación [5] se transforma en:

$$
Q_{1}^{\prime}=\sum_{\mathrm{i}=0}^{\infty}\left(\frac{y_{\mathrm{p} 2}}{1-y_{\mathrm{w} 2}} \cdot y_{\mathrm{w} 1}\right)^{\mathrm{i}} \cdot Q_{1}
$$

\section{ESTIMACIÓN DE PARÁMETROS}

A continuación se presentan algunas de las correlacionés empíricas efectuadas que relacionan datos importantes, desde el punto de vista económico, con parámetros o variables que dependen de la tecnología del proceso, pieza y condiciones del sistema.

\subsection{Tiempo de ciclo de colada}

El tiempo de ciclo de colada es el tiempo transcurrido desde que se inicia el volcado de la cuchara para llenar el cilindro inyector, hasta que se produce la separación del molde, una vez solidificada la pieza, y se extrae ésta.

En principio, se podría considerar que su valor depende del tiempo que tarde en llenarse el molde, el cual dependerá del volumen de la pieza y de la velocidad de llenado, pero en procesos de fundición inyectada, el tiempo de llenado es bastante pequeño y se puede considerar prácticamente constante el intervalo desde que se produce el volcado de la cuchara hasta que el molde se ha llenado completamente. De esta forma, la variable fundamental será el tiempo de solidificación de la colada. Sobre éste es importante resaltar que en los procesos de alta presión se efectúa tan rápido el llenado, que se puede suponer que los tiempos de llenado y de solidificación no se solapan, pudiendo encadenarlos consecutivamente para formar el tiempo total de ciclo de colada.

El tiempo de solidificación sí que varía de una a otra pieza, pudiendo obtenerse a través de su dependencia con el módulo de enfriamiento (en caso de tener varias piezas por cierre del molde, se considera el volumen y la superficie exterior total, por formar dentro del molde un único volumen unido por 
canales de llenado). Esta relación se puede expresar a través de la ecuación.

$$
t_{\mathrm{cc}}=A_{\mathrm{cc}}+B_{\mathrm{cc}}\left(\frac{V}{S_{\mathrm{ext}}}\right)^{2}
$$

La constante $B_{\text {cc }}$ depende de factores tales como el material que se está trabajando, el material del molde, características constructivas del molde, etc. La constante $A_{\mathrm{cc}}$ aparece al incluirse en $t_{\mathrm{cc}}$ el tiempo de llenado. Sus valores para una instalación analizada se pueden apreciar en la figura 4.

\subsection{Vida de los moldes}

La vida del molde metálico depende de numerosos parámetros como pueden ser el tipo de refrigeración, la continuidad en la producción, complejidad de la pieza a que da lugar, etc. Teniendo en cuenta que en la fundición a presión suele hacerse la refrigeración por agua y que el resto de parámetros tienen rangos de variación muy amplios y diversas dificultades de cara a su cuantificación, una propuesta aceptable puede ser determinar parámetros de la vida de éste por el número de partes que lo componen, $p$, que es el parámetro que permite cuantificar, de forma simple, la complejidad de la pieza. Este número de partes, según la experiencia de algunas empresas, es la principal característica que afecta a la duración del mismo. Esto es debido a que el número de defectos que presenta es mayor conforme aumenta dicho número de partes necesarias que lo constituyen, lo cual puede ser causado por el mayor número de juntas de cierre, mecanismos de asistencia, etc.

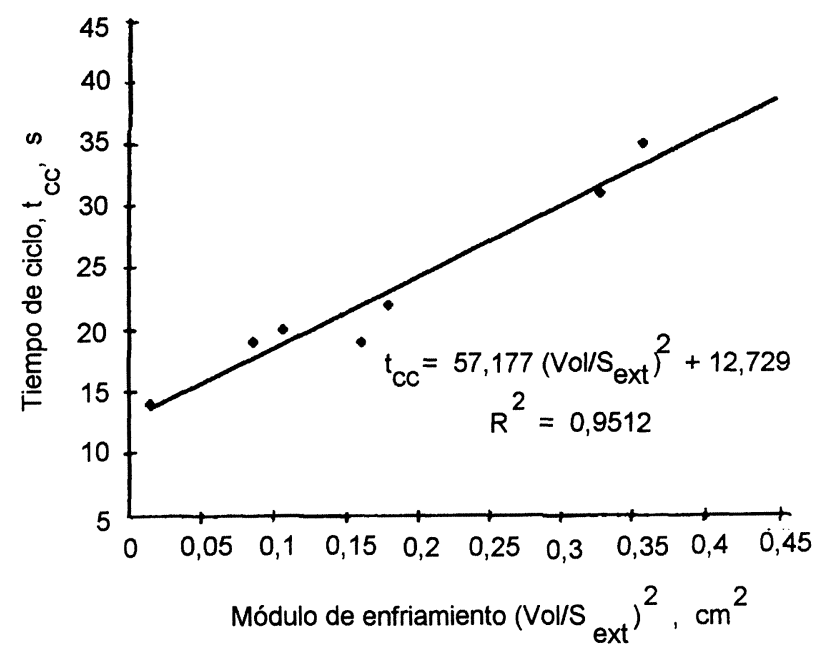

FIG. 4.- Correlación entre el tiempo del ciclo de colada y el módulo de enfriamiento.

FIG. 4.- Correlation between pouring cicle time and cooling modulus.
La experiencia adquirida en instalaciones de este tipo conduce a establecer una dependencia lineal. La vida de los moldes estará medida por el número de coladas o inyecciones efectuadas, que coincidirá con el número de piezas fabricadas, si el molde reproduce una sola pieza por cierre.

$$
V_{m}=A_{\mathrm{m}}+B_{\mathrm{m}} P
$$

En la figura 5 se dispone de una correlación para una instalación analizada.

\subsection{Rendimiento de producción de la máquina de inyección}

Con los datos obtenidos de instalaciones se puede concluir que debido a que los procesos de fundición inyectada poseen un alto grado de automatización, los parámetros que influyen se reducen y los fallos se minimizan, pudiendo considerarse que la variable que cuantifica el porcentaje de fallos es nuevamente $p$, ya que a mayor complejidad de la pieza, mayor número de partes tiene su molde y mayor número de piezas defectuosas son producidas en él.

$$
n_{p}=A_{\mathrm{p}}+B_{\mathrm{p}} p
$$

Valores de $A_{\mathrm{p}}$ y $B_{\mathrm{p}}$, al igual que en casos anteriores, pueden apreciarse en la figura 6 .

\subsection{Rendimiento másico de las piezas inyectadas}

En el estudio realizado se ha comprobado que la masa total de todas las formas auxiliares suele ser

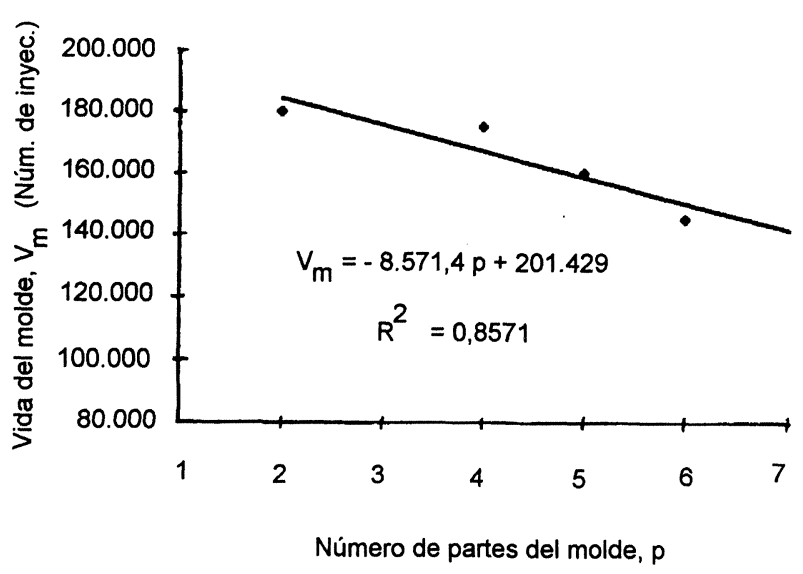

FIG. 5.- Correlación entre la vida del molde y el número de partes del mismo.

FIG. 5.- Correlation between mold life and number of parts of the same. 


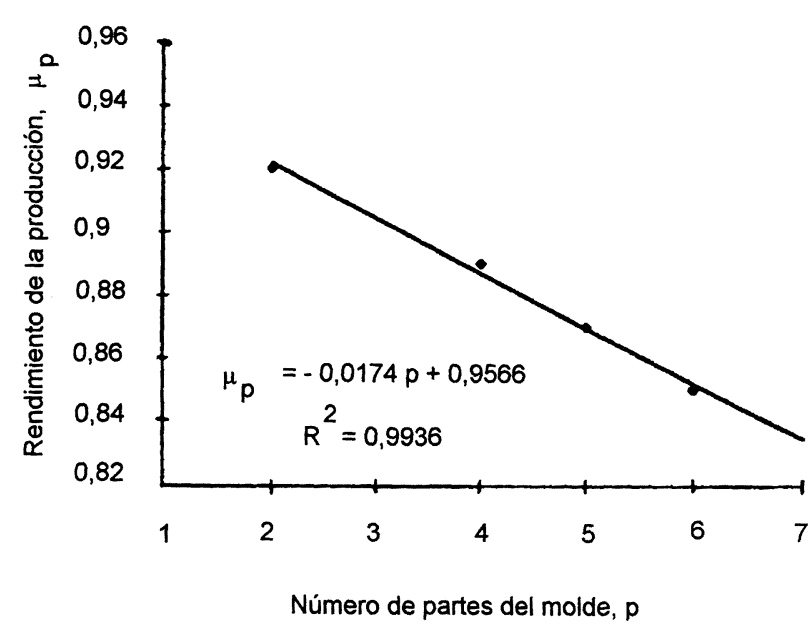

FIG. 6.- Correlación entre el rendimiento productivo y el número de partes del molde.

FIG. 6.- Correlation between yield productive and number of mold parts.

sensiblemente constante de una a otra pieza, mientras que la masa de estas, ya desbarbadas, varía en mucha mayor medida. Es por esto por lo que el rendimiento másico puede considerarse que depende del peso de la misma. Según la experiencia en instalaciones analizadas cualitativamente, el rendimiento másico varía cuadráticamente con el peso de la pieza. Por otra parte, el rendimiento también dependerá del número de piezas que el molde contiene, por lo que se tiene una familia de curvas de la forma:

$$
\mu_{\mathrm{m}}=A_{\mathrm{n}}+B_{\mathrm{n}} K+C_{\mathrm{n}} K^{2}
$$

Un ejemplo de estimación del rendimiento másico puede apreciarse en la figura 7.

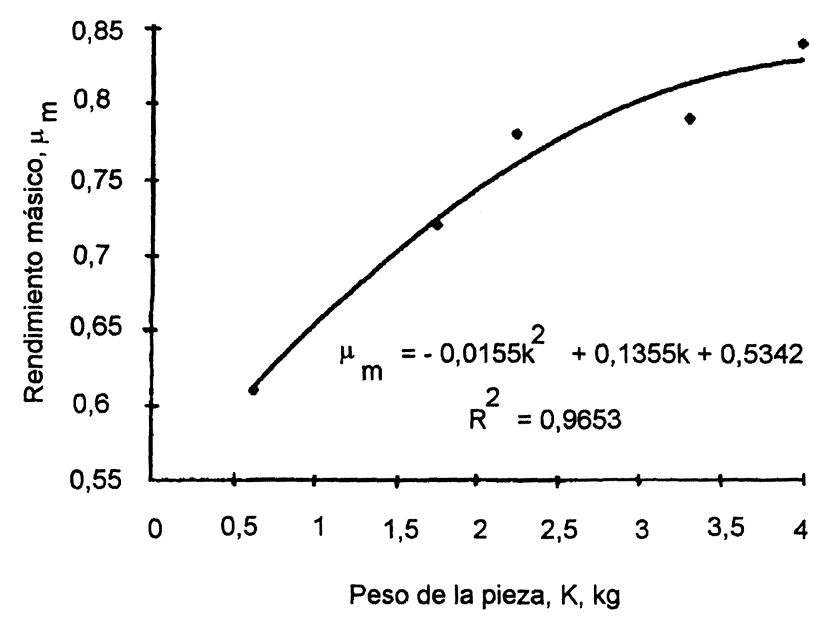

Fig. 7.- Correlación entre el rendimiento másico y el peso de la pieza.

FIG. 7.- Correlation between yield mass and workpiece weight.

\subsection{Coste de la máquina de inyección}

En función de las características geométricas de la pieza y de las condiciones de inyección, la máquina debe tener una capacidad determinada. La capacidad de la máquina es un parámetro que se evalúa a través de la fuerza de separación que en la presurización del molde es capaz de resistir la máquina. En función de la magnitud de esta fuerza, la máquina tendrá un tamaño, y por tanto, un coste determinado (12).

Para evaluar la capacidad de máquina necesitada se estima dicha magnitud en función de diferentes variables determinantes que le afecten. Las variables seleccionadas para las correlaciones con la fuerza son: peso inyectado, presión de trabajo y velocidad de inyección. Representaciones gráficas de estas correlaciones se observan en las figuras 8 , 9 y 10.

La capacidad que se tiene en cuenta para la estimación económica será la mayor de la comparación entre las tres anteriormente descritas y la calculada por el producto de la presión de trabajo por la superficie frontal fundida de la pieza. La superficie frontal fundida, se determina sumando a la superficie proyectada de la pieza en el plano de cierre del molde, la superficie de la posible mazarota y canales de alimentación.

En función de la capacidad de la máquina se estimará el coste de la misma. Esta última estimación se representa en la figura 11.

\section{EJEMPLO ILUSTRATIVO}

Los datos que han servido para la elaboración de las correlaciones y desarrollo del modelo han sido

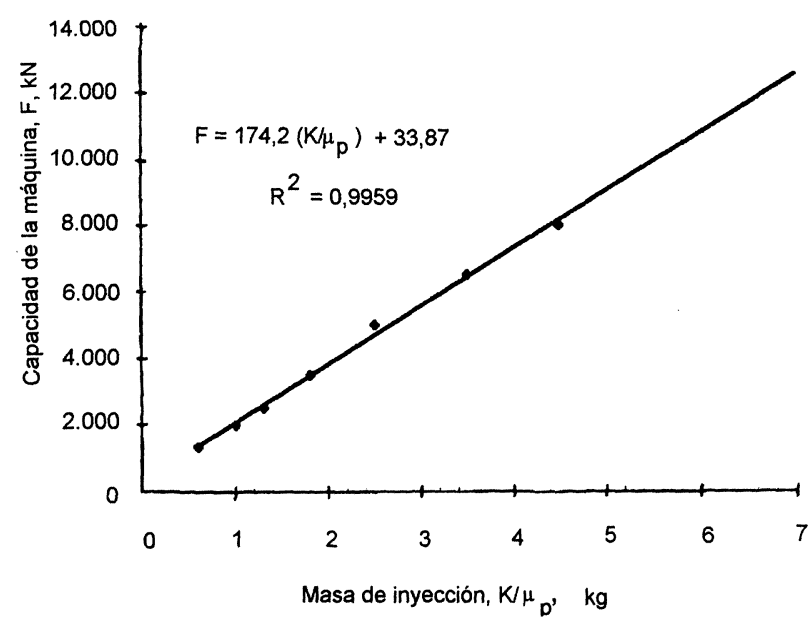

FIG. 8.- Correlación entre la capacidad de la máquina y la masa a inyectar.

FIG. 8.- Correlation between machine capacity and mass to inject. 


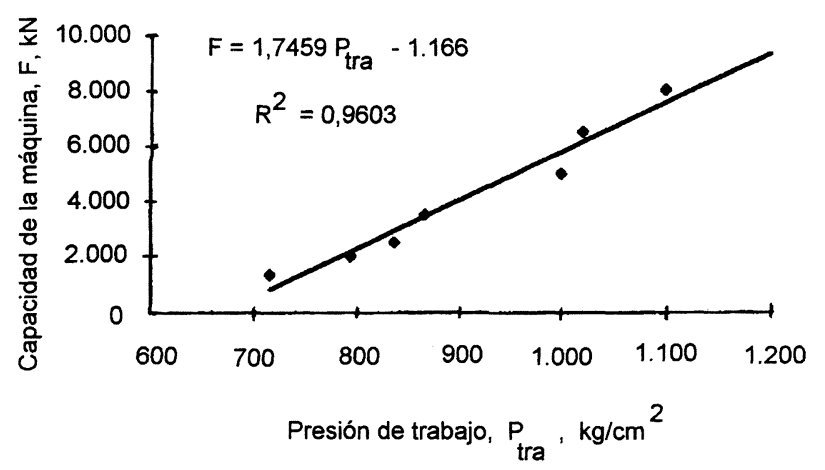

FIG. 9.- Correlación entre la capacidad de la máquina y la presión de trabajo.

FIG. 9.- Correlation between machine capacity and work pressure.

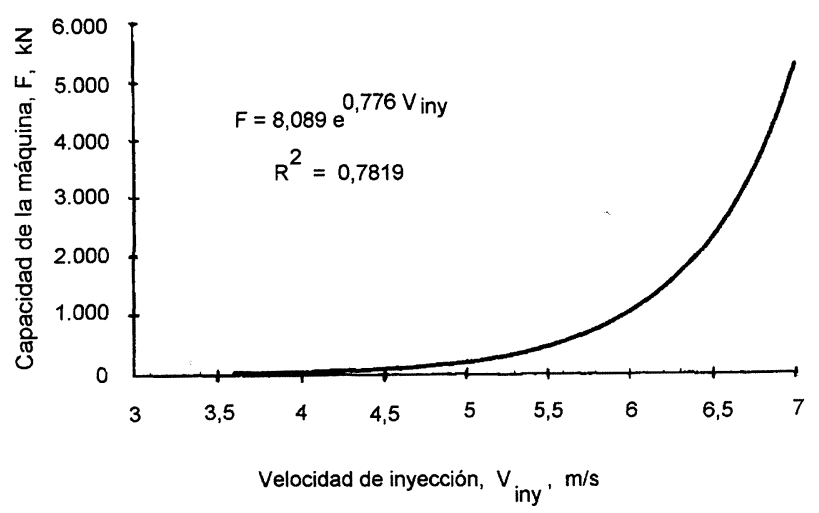

FIG. 10.- Correlación entre la capacidad de la máquina y la velocidad de inyección.

FIG. 10.- Correlation between machine capacity and inyection speed.

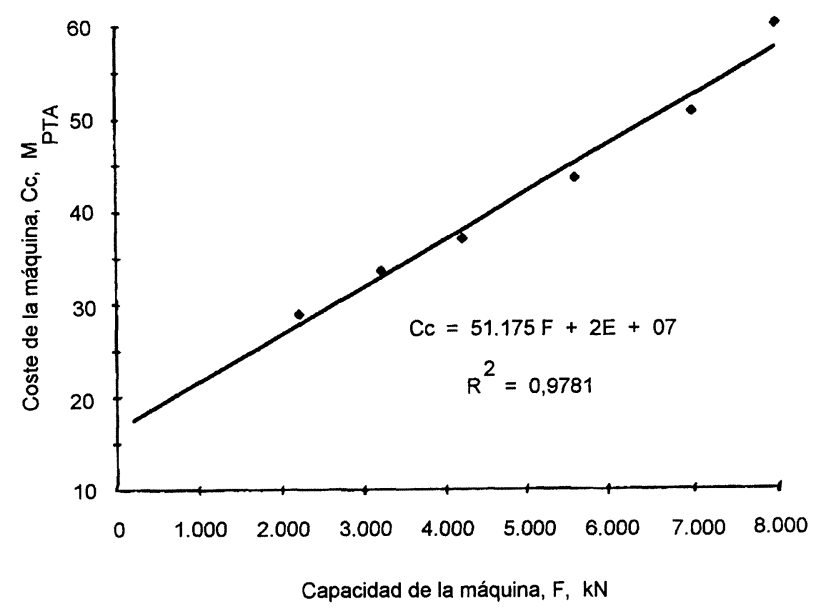

FIG. 11.- Correlación entre el coste de la máquina de inyección y la capacidad de la misma.

\section{FIG. 11.- Correlation between machine cost and} capacity of the same.

obtenidos de diversas empresas que poseen instalaciones de fundición de aluminio inyectado a alta presión. El ejemplo presentado corresponde a una instalación cuya capacidad de fusión es del orden de $1.000 \mathrm{~kg} / \mathrm{h}$. La tipología media de las piezas fabricadas responde a un peso medio de $3 \mathrm{~kg}$, y corresponden a elementos de la cadena de fabricación de vehículos automóviles tales como: tapas de cajas de cambios, cubreembragues, cárter de transferencia, etc. Dispone de unidades automáticas de alimentación, extracción y refrigeración.

En la tabla I se presentan algunos de los datos y características del sistema y pieza seleccionados para el ejemplo. Como puede apreciarse, corresponden a una pieza de pequeñas dimensiones, concretamente se trata de la tapa superior de la caja de cambios de un modelo de automóvil fabricado en la instalación. Se presentan también algunas de las estimaciones efectuadas por el modelo, así como los resultados obtenidos por el mismo. La magnitud de los resultados, evidentemente, no tiene por qué adaptarse a los valores reales de mercado, los cuales pueden presentar desviaciones sensibles de los presentados. Ello puede deberse, tanto a la política comercial de la empresa, como a las suposiciones económico-financieras introducidas en el modelo, las cuales han sido estimadas de forma orientativa no prestando una especial atención sobre ellas, ya que no son el objetivo fundamental del trabajo.

\section{RESULTADOS}

A partir del modelo descrito anteriormente y de la implementación informática realizada se dispone de una herramienta que facilita el trabajo de análisis de los parámetros más relevantes del proceso de fabricación y, por tanto, ayuda notablemente a la toma de decisiones.

A continuación, se presentan algunos resultados obtenidos en diferentes análisis de sensibilidad efectuados. Las representaciones que se muestran, constituyen algunos ejemplos interesantes que permiten analizar el entorno de fabricación de una pieza.

\subsection{Coste de fabricación unitario-volumen de producción}

A través de este análisis, se puede apreciar cómo a medida que se aumenta el volumen de producción, el coste va disminuyendo y estabilizándose en un valor determinado que dependerá del resto de entradas del modelo. Esta estabilización permite hacer predicciones cuando se compara con otras tecnologías sobre el tamaño de lote óptimo para ser competitivos.

\subsection{Coste de fabricación unitario-superficie proyectada}

Mediante este análisis se ha verificado cómo a medida que aumenta el valor de la superficie proyectada, trabajando con una misma presión de 
TABLA I.- Algunos datos, estimaciones y resultados del modelo para una pieza de aluminio de pequeño tamaño TABLE I.- Some data, estimates and outputs of the model for a small size aluminum casting

\begin{tabular}{|c|c|c|c|}
\hline Datos & \multicolumn{2}{|c|}{ Unidades } & Resultados \\
\hline Peso de la pieza & 1,38 & $\mathrm{~kg}$ & Costes variables: 512,5 PTA/pieza \\
\hline Superficie externa & 1.200 & $\mathrm{~cm}^{2}$ & \\
\hline Superficie proyectada & 338 & $\mathrm{~cm}^{2}$ & M.O. \\
\hline Volumen & 511 & $\mathrm{~cm}^{3}$ & \\
\hline Temperatura de inyección & 670 & ${ }^{\circ} \mathrm{C}$ & $\%$ \\
\hline Presión de trabajo & 650 & $\mathrm{~kg} / \mathrm{cm}^{2}$ & \\
\hline Velocidad de inyección & 3 & $\mathrm{~m} / \mathrm{s}$ & \\
\hline Núm. de turnos & 2 & & \\
\hline Horas por turno & 8 & $\mathrm{~h}$ & \\
\hline Coste material & 200 & PTA/kg & \\
\hline Coste de energía & 12,78 & $\mathrm{PTA} / \mathrm{kWh}$ & aterial \\
\hline Coste de gas & 1,6 & PTA/termia & $8 \%$ \\
\hline Amortización instalación & 10 & años & \\
\hline Núm. de partes del molde & 2 & & \\
\hline Núm. de piezas por molde & 1 & & Costes fijos: 191,7 PTA/pieza \\
\hline Temperatura de colada & 720 & ${ }^{\circ} \mathrm{C}$ & \\
\hline Rendimiento másico del horno & 0,93 & & General \\
\hline Volumen de producción & 10.000 & piezas & \\
\hline Días de ejecución & 20 & días & \\
\hline Mantenimiento & 15 & $\%$ & \\
\hline Gastos auxiliares & 25 & $\%$ & Auxiliar \\
\hline Gastos generales & 25 & $\%$ & $14 \%$ \\
\hline Trabajadores en instalación & 4 & & \\
\hline Tiempo de desbarbado & 45 & $\mathrm{~s}$ & \\
\hline Tiempo instalación/desinstalación & 2 & $\mathrm{~h}$ & \\
\hline Tiempo de reacondicionamiento & 8 & $\mathrm{~h}$ & Manten. \\
\hline Tiempo de preparación & 35 & $\min$ & $10 \%$ \\
\hline Núm. de líneas de instalación & 3 & & $31 \%$ \\
\hline Estimación & & & Coste total: 704,2 PTA/pieza \\
\hline Tiempo ciclo de colada & 23,09 & $\mathrm{~s}$ & \\
\hline Vida del molde & 184.286 & inyec. & Costes fijos \\
\hline Período de reacondicionamiento & 21.766 & piezas & \\
\hline Rendimiento productivo & 0,92 & & \\
\hline Rendimiento másico & 0,69 & & \\
\hline Coste del molde & 10.000 .000 & PTA & \\
\hline Capacidad en función de $\mathrm{P}_{\text {tra }}$ & 312,00 & $\mathrm{kN}$ & \\
\hline Capacidad en función de $V_{\text {iny }}$ & 828,80 & $\mathrm{kN}$ & \\
\hline Capacidad en función de $k / \mu \mathrm{m}$ & $3.812,80$ & $\mathrm{kN}$ & \\
\hline Capacidad en función de fuerza & $2.636,4$ & $\mathrm{kN}$ & Costes \\
\hline Coste de la máquina & 48.012 .645 & PTA & ariables \\
\hline Potencia de la máquina & 37 & $\mathrm{~kW}$ & $73 \%$ \\
\hline
\end{tabular}

inyección aumenta el valor de la fuerza de separación en el cierre del molde. Por esta razón, la capacidad requerida en la máquina de inyección aumenta, provocando al mismo tiempo que el coste de esta y de la energía que consume se incremente.

El hecho de que para valores bajos de la superficie proyectada no se produzca este incremento se debe a que, en ese rango, la capacidad de máquina no está dada por el factor fuerza de separación del cierre, siendo determinada por cualquier otro de los factores indicados anteriormente.

\subsection{Capacidad de máquina de inyección- velocidad de inyección}

De este análisis se desprende que este factor técnico no es muy determinante desde el punto de 
vista económico, debido a que hasta que no se empieza a trabajar con altas velocidades de inyección, no surgen las necesidades de maquinaria de gran capacidad.

Puesto que los rangos donde se suele trabajar en la inyección de aluminio están situados en la zona media-baja, la velocidad de inyección no es un factor determinante para la elección de la capacidad de máquina.

\subsection{Potencia de la máquina-presión de trabajo}

A medida que la presión de trabajo aumenta se necesita una mayor capacidad de máquina; por tanto, también aumenta la potencia consumida por la misma. De este análisis se desprende que la presión de trabajo no influye para valores bajos, en los que predominan otros factores como la fuerza de separación del cierre o el peso de la pieza, para la elección de la capacidad, y por consiguiente de la potencia eléctrica de la máquina a emplear en la instalación. Sin embargo, con altas presiones de inyección es este factor el que más influye, aumentando linealmente la potencia consumida con la presión de trabajo en la inyección.

\subsection{Coste energético unitario-temperatura de inyección}

En el estudio efectuado se ha verificado que los costes de energía se incrementan de una manera poco considerable con la temperatura a la que se inyecta la colada en el molde. A efectos económi$\cos$, la temperatura de inyección no afecta de manera importante, trabajando dentro del rango usualmente utilizado en este tipo de aleaciones.

\subsection{Coste de fabricación unitario-partes del molde}

En último lugar, se presenta el análisis de la sensibilidad del coste de producción unitario con respecto a la complejidad de la pieza o número de partes del molde.

En este caso se ha comprobado que el coste de fabricación unitario es bastante sensible a la variación de este parámetro, aumentando notablemente conforme lo hace la complejidad de la pieza.

\section{CONCLUSIONES}

Las conclusiones finales se pueden resumir en los siguientes puntos:
- Se ha adaptado un modelo técnico-económico al proceso de fundición inyectada a alta presión, que permite explorar los parámetros técnicos o variables físicas fundamentales, que pueden ser más determinantes a la hora de analizar económicamente el proceso.

- Los resultados obtenidos han sido verificados experimentalmente en distintas instalaciones industriales ajustándose de forma satisfactoria a la experiencia del sector productivo.

- Se ha efectuado una implementación informática del modelo propuesto de manera que la utilización del mismo resulta más cómoda y sencilla, debido en gran medida al entorno de programación empleado. Esto ha permitido efectuar análisis de sensibilidad rápidos y fiables.

- De los estudios de sensibilidad realizados se desprende que existen algunos factores técnicos que tienen poca relevancia desde la perspectiva de un análisis económico, pues una gran variación de estos no provoca una similar en las salidas económicas a las que afecta. Tal es el caso de la temperatura o la velocidad de inyección que, sin embargo, son importantísimos desde la perspectiva física del proceso. Otras variables o parámetros sí que afectan de una manera mucho más determinante. Tal es el caso de parámetros como el número de partes del molde o la superficie proyectada de la pieza, que con pequeñas variaciones afectan de una manera notable al coste de fabricación del producto.

\section{Agradecimientos}

El autor quiere expresar su agradecimiento a las empresas Suzuki, Toyota, Bosh y UBE Industries, por la ayuda, interés y gran cantidad de información suministrada. Asimismo, se agradece al Comité Científico de la OTAN la beca concedida para el desarrollo del Proyecto de Investigación, por el cual se ha desarrollado, en parte, este trabajo, en el Massachusetts Institute of Technology, MIT. EE.UU..

\section{Notación}

$\mu_{\mathrm{m}} \quad$ Rendimiento másico.

$\mu_{\mathrm{p}} \quad$ Rendimiento productivo.

$F \quad$ Capacidad de la máquina en función de algún parámetro.

$K \quad$ Peso de la pieza.

$p \quad$ Número de partes del molde.

$P_{\text {tra }} \quad$ Presión de trabajo.

$P_{\mathrm{j}} \quad$ Paso $j$ del proceso.

$q_{\mathrm{j}} \quad$ Salida requerida por el volumen de producción objetivo para $P_{\mathrm{j}}$. 
$Q_{\mathrm{j}} \quad$ Entrada para el $P_{\mathrm{j}}$.

$Q^{\prime} \quad$ Entrada para el $P_{\mathrm{j}}$ incluyendo la salida reciclable del paso $j$ del proceso.

$S_{\text {ext }} \quad$ Superficie externa.

$S_{\text {pro }}$ Superficie proyectada.

$t_{\mathrm{cc}} \quad$ Tiempo del ciclo de colada.

$V_{\text {iny }}$ Velocidad de inyección.

$V_{\mathrm{m}} \quad$ Vida del molde.

Vol Volumen de la pieza.

$V P \quad$ Volumen de producción.

$W_{\mathrm{j}} \quad$ Salida reciclable para el $P_{\mathrm{j}}$.

$X_{\mathrm{j}} \quad$ Salida no recuperable para el $P_{\mathrm{j}}$ y para el proceso principal.

$y_{\mathrm{ij}} \quad$ Rendimiendo relativo del $P_{\mathrm{j}}$ para la salida $i$, siendo $i: P, W \circ X$.

\section{REFERENCIAS}

(1) Szekely, J y Trapaga, G. J. Mat. Res., 10 (9), 1995: 2.178-2.196.
(2) Dieffenbach, J.R. y Mascarin, A. J. Mater., 3 (45), 1993: 16-19.

(3) Mangin, G.E., NeEly, J.E. III y ClaRK, J.P., J. Mater., 3 (45), 1993: 23-27.

(4) Mariano, S.A., Tuler, F.R. y Owen, W.S. J. Mater., 3 (45), 1993: 20-22.

(5) Puttre, M., Mech. Eng., 1993: 69-71.

(6) Ostermann, F. et al., Aluminium Materials Technology for Automobile Construction. Mechanical Engineering Publ. Ltd., Londres (R.U.), 1993.

(7) Winter, D. Ward's Automotive Yearbook, 1994: 23-27.

(8) Auto Strategies International, World Automotive Market Report, 1994: 17.

(9) American Society for Material, Metals Handbook, vol. 15: Casting, ASM Int., Columbus, OH (EE.UU.), 1988: 294-295.

(10) American Foundrymen's Society, Aluminum Casting Technology, $2^{a}$ ed., AFS inc., Des Plaines, IL (EE.UU.), 1993: 145-166.

(11) Faura, F., Rev. Met. Madrid, 32 (6), 1996: 381-390.

(12) American Foundrymen's Society, Casting Buyer's Guide, $1^{\text {a }}$ ed, AFS inc., Des Plaines, IL (EE.UU.), 1988: 44-64. 